\title{
Supporting information for Ultrasensitive Tip- and Antenna-Enhanced Infrared Nanoscopy of Protein Complexes
}

\author{
Brian T. O'Callahan ${ }^{1}$, Mario Hentschel ${ }^{2}$, Markus B. Raschke ${ }^{3}$, Patrick Z. El-Khoury ${ }^{1}$, and A. Scott Lea ${ }^{1}$ \\ ${ }^{1}$ Enviromental Molecular Sciences Laboratory, Pacific Northwest National Laboratory, Richland, WA 99352, USA \\ ${ }^{2} 4^{\text {th }}$ Physics Institute, Research Center SCoPE, and Center for Integrated Quantum Optics, IQST University of \\ Stuttgart, Pfaffenwaldring 57, 70569 Stuttgart, Germany \\ ${ }^{3}$ Department of Physics, Department of Chemistry, and JILA, University of Colorado at Boulder, Boulder, CO, \\ 80309 USA
}


Fig. S1 shows FTIR transmission spectra of the ferritin-coated antennas, acquired using a commercial FTIR microscope (IFS 66/s, Bruker) with a 15x, 0.4 NA objective with a diffraction-limited spot size diameter of $\sim 14 \mu \mathrm{m}$, so that $\sim 100$ antennas are in the field of view. The antenna were arranged in arrays with $2.5 \mu \mathrm{m}$ spacing in both lateral dimensions, over a $200 \mu \mathrm{m}$ by $200 \mu \mathrm{m}$ square, creating 6400 antenna in total. The antenna resonance frequency red-shifts with increasing antenna length, and small Fano spectral lineshapes appear around $1540 \mathrm{~cm}^{-1}$ and $1660 \mathrm{~cm}^{-1}$ due to the amide II and amide I responses of the protein, respectively. Fits to the coupled harmonic oscillator model (Eq. 2) of the main text are shown as solid lines.

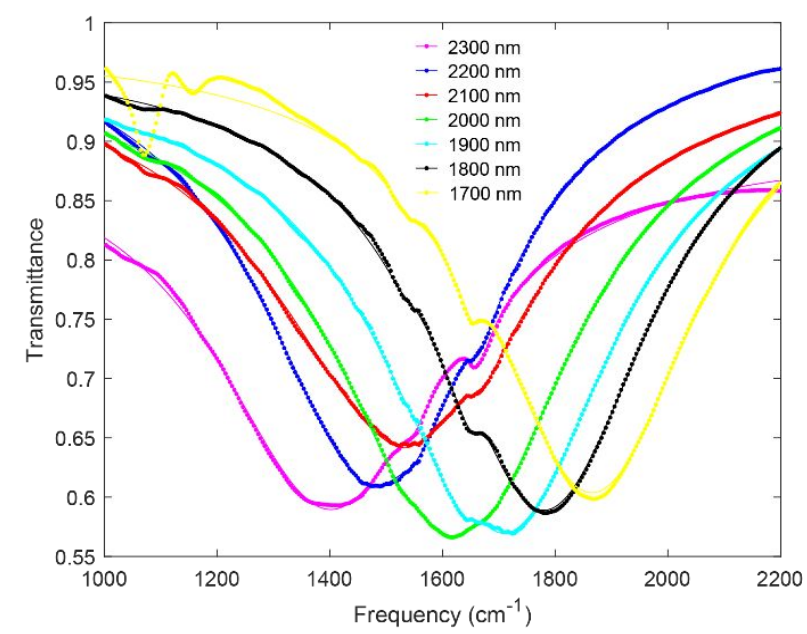

Figure S1. Far-field FTIR transmission spectra of the ferritin-coated antenna arrays. The plasmonic antennas show broad resonances spanning the amide region. Small modulations at $\bar{v}=1660 \mathrm{~cm}^{-1}$ and $1540 \mathrm{~cm}^{-1}$ are the protein amide I and amide II spectral response, respectively. Solid lines indicate fits to Eq. 2 of the main text.

Here, we outline the normalization procedure applied to each hyperspectral dataset shown here and in the main text. First, magnitude and phase images were acquired using the standard pseudoheterodyne technique. Then the magnitude images $A(x, y)$ were divided by the mean value within a selected reference region and the phase images $\Phi(x, y)$ were subtracted by the corresponding phase value. The data was then converted to a complex number using $\tilde{E}_{\text {scat }}(x, y)=A(x, y) \exp (i \Phi(x, y))$. 

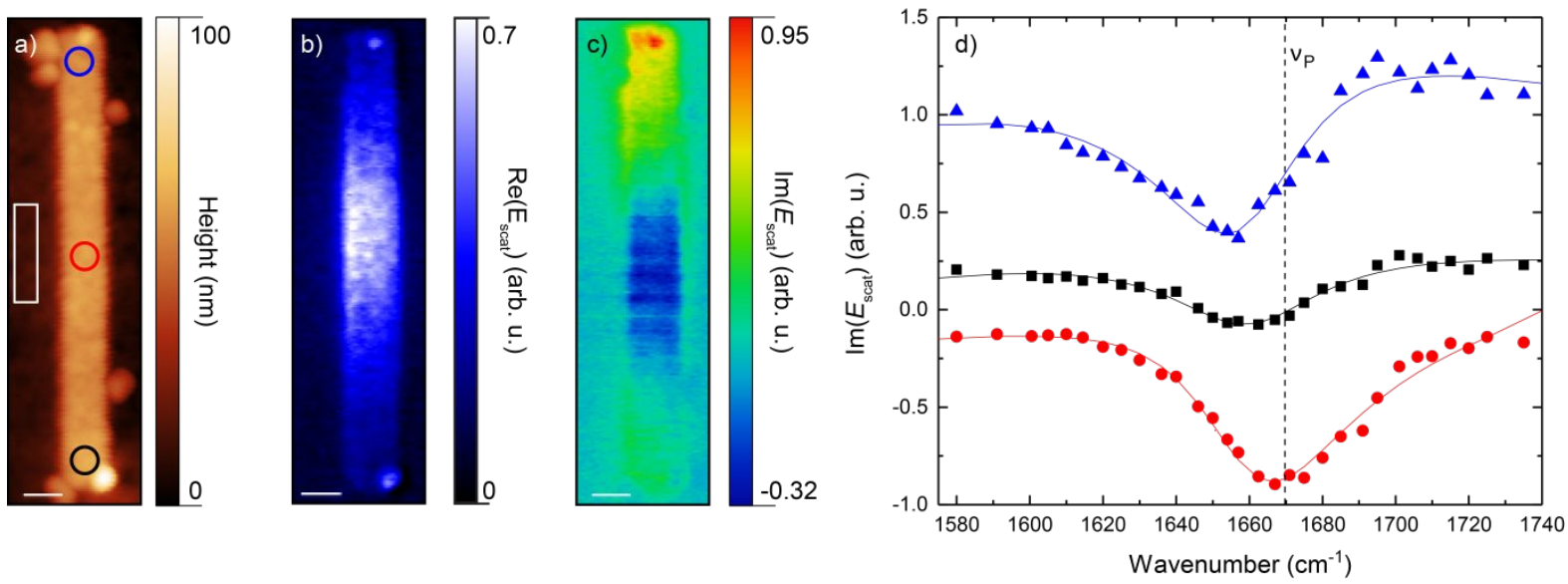

Figure S2a) Topography and b) real and c) imaginary part of $E_{\text {scat }}$ of an $\mathrm{L}=2100 \mathrm{~nm}$ long ferritin-coated antenna acquired at $\bar{v}=1667 \mathrm{~cm}^{-1}$ (scalebar is $200 \mathrm{~nm}$ ). d) Point spectra acquired at positions indicated by the correspondingly colored points in a). Spectra are normalized to a ferritin $/ \mathrm{CaF}_{2}$ region indicated by the white rectangle in a). A small, $\sim 10 \mathrm{~nm}$ high inhomogeneity is present at the top end of the antenna, which is highlighted in Fig. 4 of the main text.

In Fig. S2, we show the a) topography, b) near-field optical image at $\bar{v}=1667 \mathrm{~cm}^{-1}$, and c) point spectra from corresponding points in a) of a ferritin-coated antenna with length $\mathrm{L}=2.1 \mu \mathrm{m}$. A spectrum from the ferritin/ $\mathrm{CaF}_{2}$ region indicated by the white rectangle in a) was used as a reference to normalized the spectra in c). A small $\sim 10 \mathrm{~nm}$ high protein aggregate is present at the top end of the antenna, which is shown in Fig. 4 of the main text, and is used to analyze our sensitivity limits.

Fig. S3 shows the spatio-spectral results from a $\mathrm{L}=2100 \mathrm{~nm}$ coated nanoantenna at the edge of
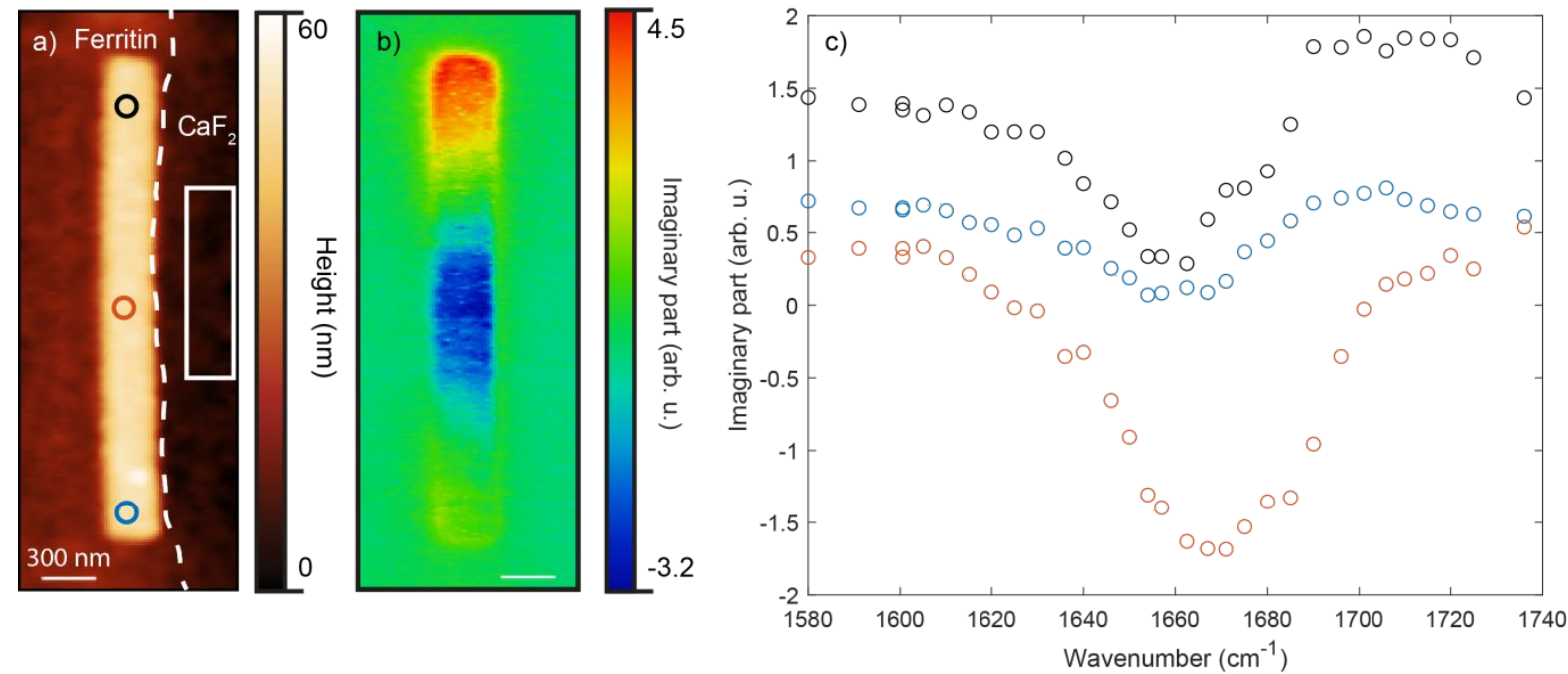

Figure S3a) Topography of antenna at the boundary of the ferritin layer. The ferritin layer is distinguished by a $\sim 10 \mathrm{~nm}$ step height. White solid rectangle represents the reference region. b) Corresponding near-field image of antenna under resonant laser excitation. c) Point spectra extracted from regions indicated by correspondingly colored circles in a). The spectra qualitatively match those presented in Fig. S2. 

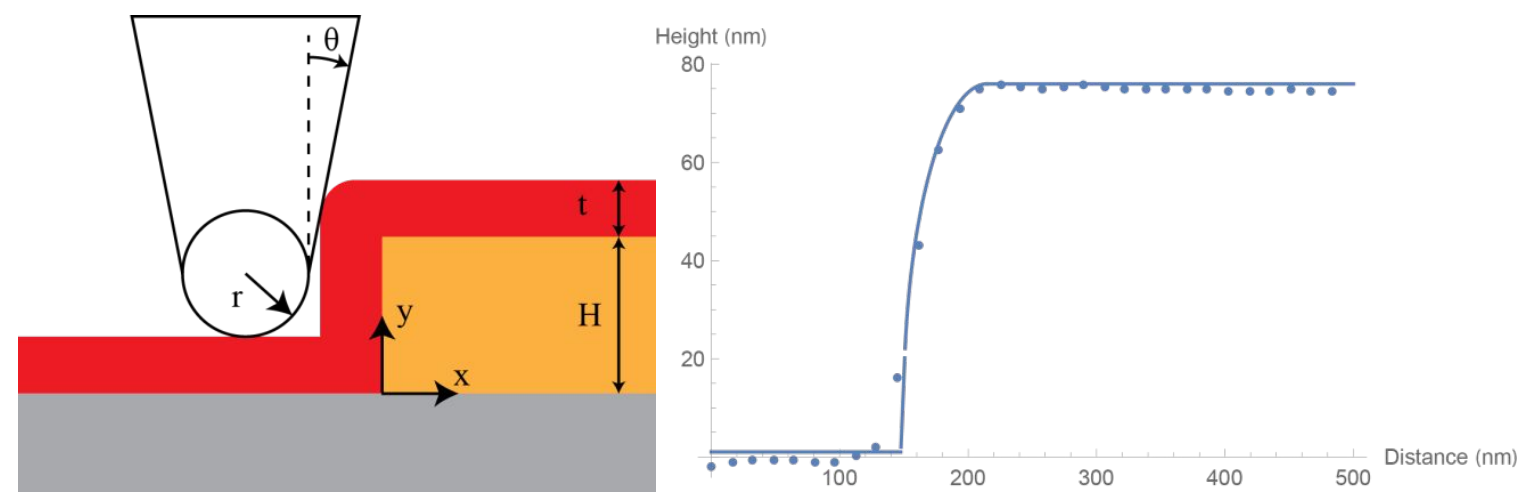

Figure S4. a) Schematic to extract tip apex radius from topography linecut across antenna edge. Sample is modelled as a step-height of height $H$, covered by a ferritin layer of thickness $t$. The tip is modelled as a conical taper with angle $\theta$ and apex radius of curvature $r$. b) Topography linecut and fit across an antenna step edge using a tip radius of $r=50 \mathrm{~nm}$.

the spin-coated ferritin layer. The spectra in c) are normalized to the bare $\mathrm{CaF}_{2}$ surface, and qualitatively match the spectra presented in Fig. S2c) above. The close agreement between the spectra in Figs. S2c) and $\mathrm{S} 3 \mathrm{c}$ ) indicate that normalizing to a ferritin/ $\mathrm{CaF}_{2}$ region does not significantly impact the spectral lineshapes.

A schematic of the tip and sample geometry used in our tip-scan model is shown in Fig. S4a). The antenna is modelled as a step-edge of height $\mathrm{H}$, and the ferritin coating is modelled with a smooth $12 \mathrm{~nm}$ film, creating a rounded edge. The tip shaft angle of $\theta=9$ degrees is taken from the manufacturers' specifications, and the tip apex radius $r$ is used as a fit parameter. A linecut along the antenna edge is fit to this model with an apex radius of $50 \mathrm{~nm}$ (Fig. S4b).

Figure S5 shows the a) topography and b) nanospectrum of a ferritin sample spin-coated on a uniform, unpatterened template-stripped gold surface. The spectrum was extracted by averaging the spectra within the blue boxed region in Fig. S5a), and was normalized to the gold surface. The thickness of this region is $\sim 40 \mathrm{~nm}$, so that the entire film thickness is within the probing depth of the tip (approximately given by the tip apex radius). The spectrum is fit to a two Lorentzian curve, which incorporates the amide I and amide II peaks at $1667 \mathrm{~cm}^{-1}$ and $1550 \mathrm{~cm}^{-1}$, respectively. The calculated NSNR for this spectrum is $0.63 \sqrt{\mathrm{Hz}} / \mathrm{cm}^{-1}$, indicating an order of magnitude lower sensitivity than that obtained using the resonant nanoantenna.

Figure S6 shows the dependence of the coupled oscillator model on a) increasing molecular phase 

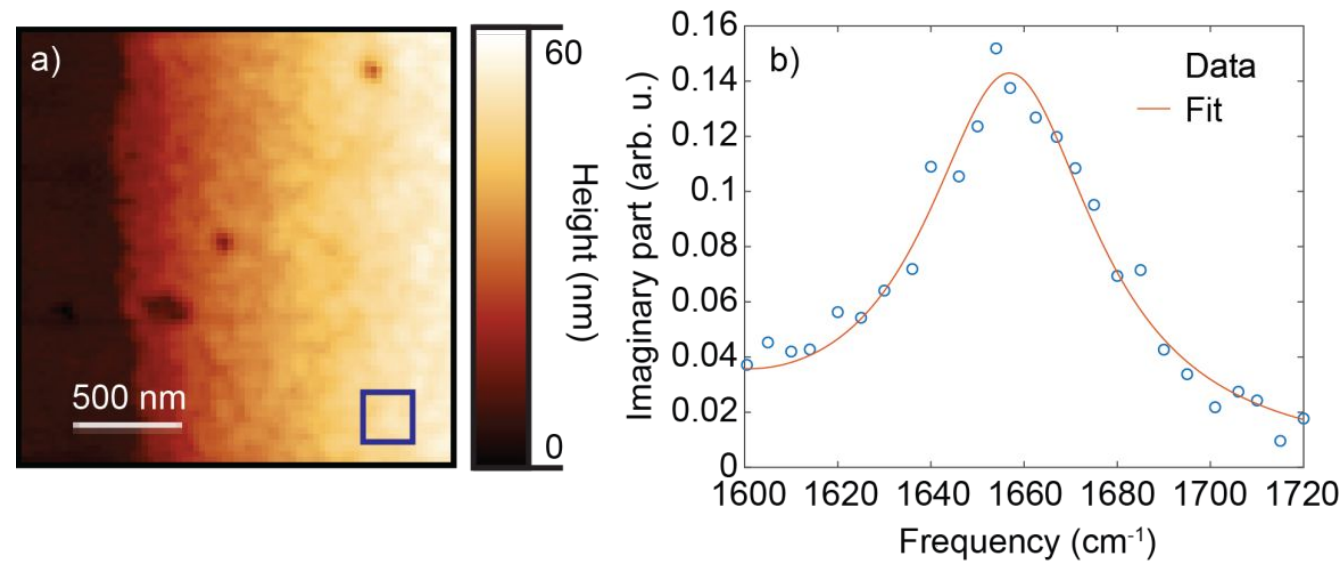

Figure S5. Spin-coated ferritin on template stripped gold substrate. a) Topography near the edge of the ferritin layer. b) s-SNOM spectrum of the area indicated by the blue box in a)

and b) coupling strength. The degree of asymmetry is determined primarily by the molecular phase, with a slight contribution from the coupling strength.

Figure S7 shows a helium ion microscope image of an uncoated antenna, displaying the roughness of the gold surface. 
Here, we provide additional experimental details of our $s$-SNOM studies. The tip-scattered light

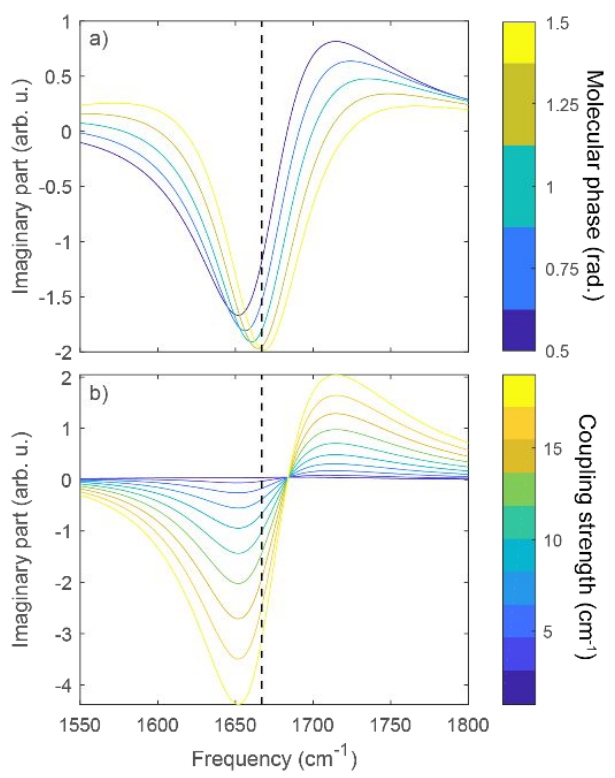

Figure S6. Dependence of model on the molecular phase, and the coupling strength. Degree of asymmetry is determined by the phase of the molecule field, and to a lesser extent the coupling strength. 
was detected by a mercury-cadmium-telluride detector (MCT, KLD-0.25-J1/11/DC5M, Kolmar 


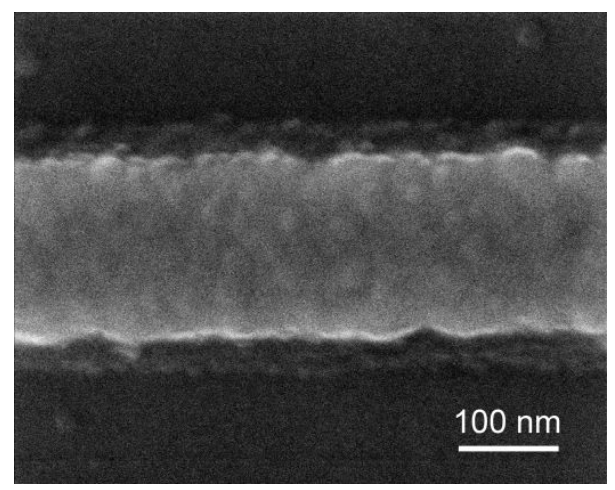

Figure S7. Helium ion microscopy image of an uncoated antenna showing the morphology of the bare gold surface.

Technologies). The MCT signal was demodulated at the third harmonic of the tapping frequency to remove the far-field background using a lock-in amplifier (HF2LI, Zurich instruments). Approximately $4 \mathrm{~mW}$ of QCL power (FWHM bandwidth $<100 \mathrm{MHz}$ ) was focused with an off-axis parabolic mirror with effective focal length $\mathrm{f}=25.4 \mathrm{~mm}$ (numerical aperture $\mathrm{NA}=0.44$ ) to yield $2 \mathrm{~kW} / \mathrm{cm}^{2}$ intensity at the tip apex. We used $\sim 80 \mathrm{~nm}$ peak-to-peak tip tapping amplitude. Incident laser light was s-polarized along the long axis of the antenna to excite the plasmonic modes. A quarter-waveplate was used in the reference arm to rotate the field by $90^{\circ}$ so that the reference field effectively interferes with the vertically-polarized tip-scattered field. The spatial resolution of our optical images was $\sim 50 \mathrm{~nm}$, matching the tip apex radius of curvature.
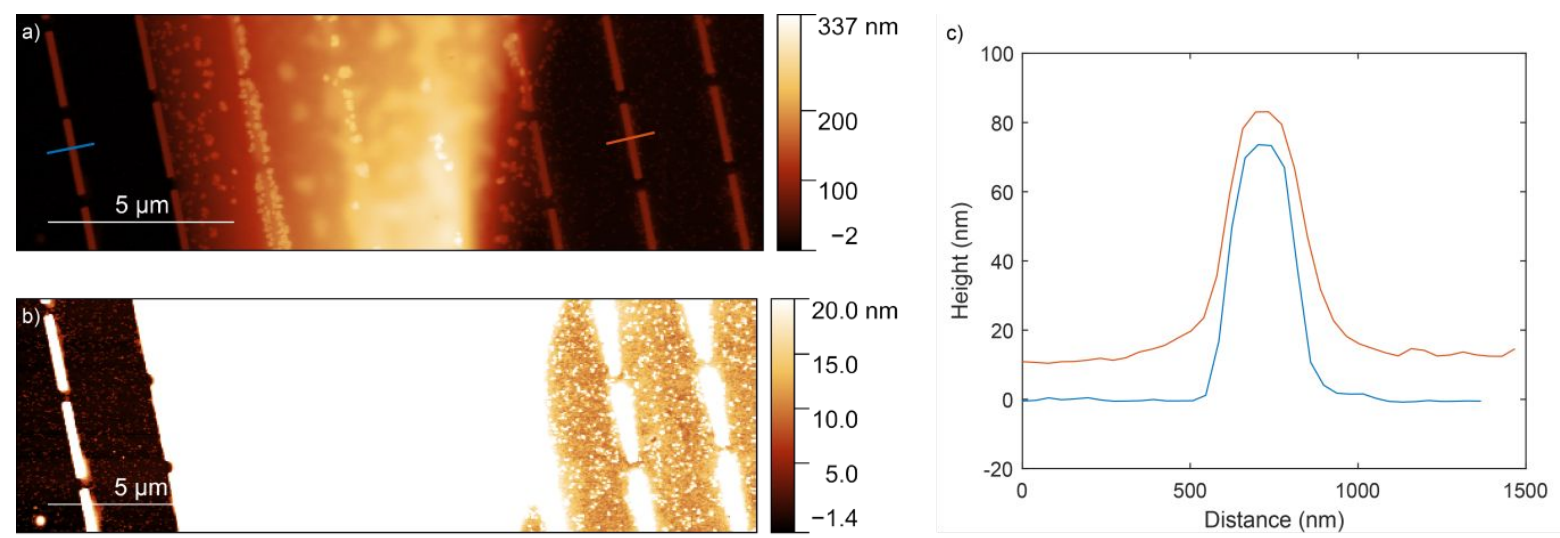

Figure S8a) AFM topography at the edge of the spin-coated ferritin layer. While there is a large aggregate at the layer boundary, a smooth monolayer is present on the antenna on the right side of the image. b) Same image as a) with increased contrast to display the monolayer coating. c) Linecuts along corresponding lines in a) showing a $10 \mathrm{~nm}$ coating thickness. 
Ferritin was deposited via spin coating $10 \mu \mathrm{L}$ of an aqueous $12 \mu \mathrm{M}$ protein solution at $3000 \mathrm{RPM}$ for 20s. Fig. S8a) shows an AFM image across the edge of the ferritin layer. While there is a thick section aggregate at the edge of the layer in the center of the image due to aggregation during the spin coating process, a continuous, $\sim 12 \mathrm{~nm}$ thick layer exists on the antennas within the layer boundary. The $12 \mathrm{~nm}$ height is consistent with the ferritin diameter value, indicating that the film is predominately monolayer. Occasional bilayer regions are observed in Fig. S8b). Fig. S8c) shows linecuts across coated (red curve) and uncoated antenna (blue curve), which indicate that the monolayer film persists on the antenna surface.

\begin{tabular}{|l|l|l|l|l|l|l|l|}
\hline Antenna length $(\mu \mathrm{m})$ & 1.7 & 1.8 & 1.9 & 2.0 & 2.1 & 2.2 & 2.3 \\
\hline Resonant frequency $\left(\mathrm{cm}^{-1}\right)$ & 1880 & 1800 & 1730 & 1650 & 1570 & 1510 & 1420 \\
\hline Linewidth $\left(\mathrm{cm}^{-1}\right)$ & 460 & 480 & 510 & 530 & 570 & 500 & 500 \\
\hline
\end{tabular}

Table 1. Resonant properties of ferritin-coated nanoantennas extracted from fits to the far-field spectra in Fig. S1.

\begin{tabular}{|l|l|l|}
\hline & Resonance frequency $\left(\mathrm{cm}^{-1}\right)$ & Linewidth $\left(\mathrm{cm}^{-1}\right)$ \\
\hline Amide I & 1660 & 56 \\
\hline Amide II & 1540 & 60 \\
\hline
\end{tabular}


The topography and optical image of an uncoated antenna is shown in Figs. S9a) and b), respectively. The optical response is smoothly varying, with no significant topographic artifacts, as evidenced by the linecut shown in panel c).
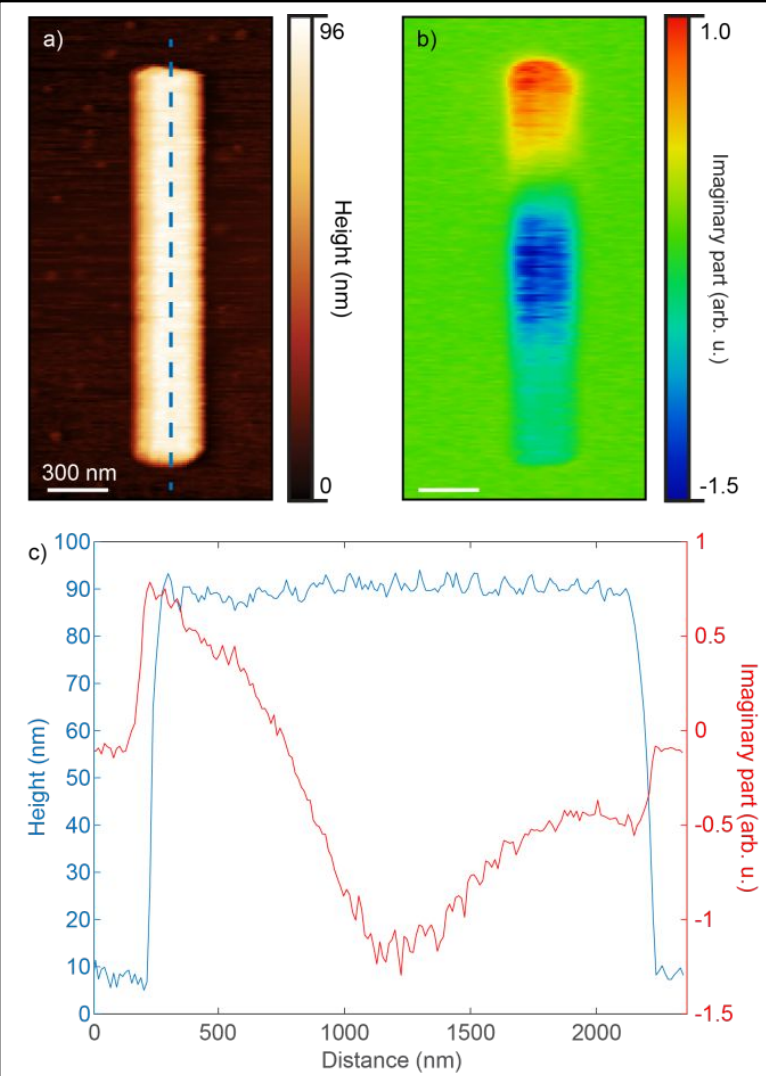

Figure S9. a) Topography and b) near-field image of an uncoated antenna acquired at $\bar{v}=1736 \mathrm{~cm}^{-1}$. c) Linecut of topography (blue) and optical signal (red) along antenna length showing the smoothly varying spatial response of the antenna in the absence of a heterogeneous protein film. 